\title{
Preparation of Polythiophene Copolymer for Third Order Nonlinear Optics
}

\author{
Haruki OKawa, Tatsuo Wada, and Hiroyuki SaSabe \\ Frontier Research Program, The Institute of Physical and Chemical Research (RIKEN), \\ Hirosawa 2-1, Wako, Saitama 351-01, Japan
}

(Received August 15, 1996)

\begin{abstract}
KEY WORDS Polythiophene / Copolymer /Nonlinear Optical Property / Optical Third Harmonic Generation / $\pi$-Conjugation /
\end{abstract}

Organic third order nonlinear optical materials have been investigated for potential applications in optical devices such as all-optical switches, logic systems, and light-modulators. ${ }^{1-3}$ In particular, one-dimensional conjugated polymers such as polyacetylene, polydiacetylene have a very large and fast third-order nonlinear optical effect. $^{4-6}$ In these one-dimensional systems, the nonlinear response is derived from a large dipolar transition of $\pi$-electrons delocalized along their main chains. Consequently, the macroscopic nonlinear susceptibility $\chi^{(3)}$ $\left(-\omega_{1} ; \omega_{2}, \omega_{3}, \omega_{4}\right)$ is expressed as,

$$
\chi^{(3)}\left(-\omega_{1} ; \omega_{2}, \omega_{3}, \omega_{4}\right)=N f_{\omega_{1}} f_{\omega_{2}} f_{\omega_{3}} f_{\omega_{4}}\langle\gamma\rangle
$$

and for non-oriented systems as,

$$
\langle\gamma\rangle=\frac{1}{5} \gamma_{x x x x}
$$

where $N$ is the density of nonlinear active chromophore, the $f$ 's are the local field factors for each frequency $\left(\omega_{i}\right)$, and $\gamma_{x x x x}$ is the microscopic hyperpolarizability along the conjugated polymer main chains.

After the synthesis of polyacetylene was reported, many polymers have been synthesized because of their desirable electronic properties. However, these polymers of the expanded $\pi$-electron systems have very poor processability and bad linear optical qualities (light scattering and long cut-off wavelength) due to the interaction between the main chains, and are thus are not suitable for optical device applications. Although the substitution of the bulky side groups to the main chain improves their processability, it causes a reduction of $\pi$-conjugation in the main chain and a dilution of the concentration of the nonlinear optical active chromophore. While these changes do lead to somewhat of a decrease of the nonlinear optical properties they enable the fabrication of the nonlinear waveguide structures with long enough interaction lengths to offset the reduction of the nonlinearity.

Poly(3-alkylthiophene) is one of these soluble onedimensionally conjugated polymers, ${ }^{7-9}$ and there have many studies of both its linear and nonlinear optical properties. ${ }^{10-11}$ The expansion of $\pi$-conjugation in the main chain is found to enhance the hyperpolarizability $\left(\gamma_{x x x x}\right.$ in eq 1$),{ }^{12}$ and if the length of the side chains are long enough ( $<$ hexyl), the change of the alkyl chain has a negligible affect on the $\pi$-conjugation. ${ }^{11}$ Mechanical stretching of the polymer films aligns the polymer main chain and expands the $\pi$-conjugation, ${ }^{13}$ and this further enhance the value of $\left\langle\gamma_{x x x x}\right\rangle$. The nonlinear optical responses were found to be ultra-fast (less than one picosecond) by degenerate four wave mixing and interferometric measurements. ${ }^{14-16}$ The excellent linear optical and mechanical properties of these polymers has enabled the fabrication of polymer waveguides in which nonlinear refractive index changes were observed. ${ }^{11}$ The density, $N$, of nonlinear optical (NLO) active sites is also an important factor for macroscopic susceptibility $\chi^{(3)}$. Copolymerization of thiophene monomers with long and short side chains is a convenient method to change the value of $N$ in a continuous manner. In this study, we investigated the effects of copolymerization on the $\pi$ conjugation and tried to improve the nonlinear optical properties of the thiophene copolymer.

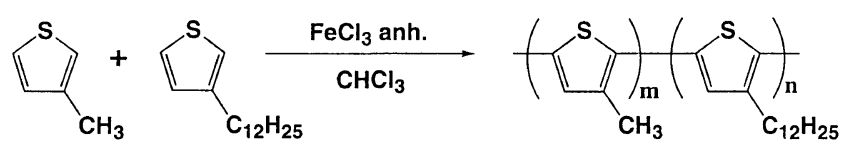

Scheme 1. Synthesis of polythiophene copolymer.

\section{EXPERIMENTAL}

\section{Monomers}

Monomers, 3-dodecyl and 3-methylthiophene, were obtained from Tokyo Kasei Kogyo Co. and purified by vacuum distillation. Chloroform for polymerization was purified by standard procedures and used immediately.

\section{Polymerization}

The copolymerization was carried out using the following procedure. ${ }^{7}$ The monomers (total amount $10 \mathrm{mmol}$ ) were dissolved in $20 \mathrm{ml}$ of dry chloroform. Anhydrous $\mathrm{FeCl}_{3}$ was added to the solution and stirred for $15 \mathrm{~min}$. The reaction mixture was poured into $100 \mathrm{ml}$ of methanol and the crude copolymer was isolated by filtration. The ionic impurities were removed by soxhlet extraction with methanol for 3 days. Following this the dedoped polymer was purified three times by reprecipitation from chloroform to methanol and dried under vacuum.

\section{Measurement}

${ }^{1} \mathrm{H}$ NMR spectra and UV-VIS absorption spectra of the copolymers were recorded by using JEOL GX500 FT-NMR and Shimadzu UV-3100 spectrophotometers, respectively. The third-order nonlinear optical susceptibility $\chi_{1111}^{(3)}(-3 \omega ; \omega, \omega, \omega)$ of the copolymer was deter- 
mined by optical third harmonic generation (THG) measurement using a fundamental of $1.907 \mu \mathrm{m} .{ }^{17} \chi^{(3)}=$ $1.4 \times 10^{-14}$ esu for fused silica was used as a reference.

\section{RESULTS AND DISCUSSION}

Table I summarize the results of copolymerization. The conversion percentage of the copolymers was calculated according to,

$$
\text { Conversion }(\%)=\frac{\text { Weight of the obtained copolymer }}{\text { Total weight of the comonomers }}
$$

Following the copolymerization, a small insoluble amount remained which was removed by reprecipitation. This part was not included in the conversion. It was found that when more than $60 \% 3$-methylthiophene was used the copolymerization yielded only an insoluble mass. Also, longer polymerization times resulted in larger insoluble fractions and reduced the conversions. In Table I, the conversions of the copolymerization were higher than $50 \%$. However as the fraction of 3-methylthiophene was increased the molecular weight of the copolymers became lower and the distribution of the molecular weight broadened. The copolymer was precipitated as a doped polymer during the polymerization. The molecular weight and its distribution were dominated by the solubility of the obtained copolymer. As the fraction of 3-methylthiophene monomer was increased, the solubility of the copolymer became poorer and the polymer precipitated out at earlier stages of polymerization. The precipitation causes a reduction of the molecular weight and a broadening of the molecular weight distribution.

To characterize the structure of the copolymer, the ${ }^{1} \mathrm{H}$ NMR spectra were measured (Figure 1). The peaks are assigned to the methyl proton of the dodecyl group (at $0.92 \mathrm{ppm}$ ), the methylene proton (at $1.28,1.68 \mathrm{ppm}$ ), and the aromatic proton of the thiophene ring (at $6.98 \mathrm{ppm}$ ). The peaks at $\delta=2.82$ and $2.53 \mathrm{ppm}$ correspond to the $\alpha$-methylene proton of the dodecyl group for head-to-tail and head-to-head coupling, respectively. ${ }^{18}$ The signal of the methyl proton of 3-methylthiophene unit is also observed to split into two peaks (at 2.43 and $2.23 \mathrm{ppm}$ ) which may be attributed to those for the two kinds of coupling as well as the signals of the $\alpha$-methyl proton. From the intensity of the signals, the ratio of the head-to-head coupling for 3-methylthiophene unit is determined to be $10 \%$. This value is almost the same as

Table I. Copolymerization of 3-dodecylthiophene and 3-methylthiophene ${ }^{\mathrm{a}}$

\begin{tabular}{cccc}
\hline $\begin{array}{c}\text { Methyl fraction } \\
\text { in seed/mol\% }\end{array}$ & \begin{tabular}{c} 
Conversion $^{\text {b }}$ \\
\cline { 2 - 3 }
\end{tabular} & $\begin{array}{c}\text { Methyl fraction } \\
\text { in polymer/mol\% }\end{array}$ & $\begin{array}{c}\bar{M}_{n}{ }^{\mathrm{c}} \\
\times 10^{4}\end{array}$ \\
\hline 60 & 27.8 & 52.0 & 2.4 \\
50 & 32.9 & 46.8 & 2.5 \\
40 & 35.3 & 40.0 & 2.7 \\
30 & 53.3 & 34.3 & 2.6 \\
20 & 52.1 & 28.2 & 2.7 \\
10 & 53.4 & 12.0 & 2.8 \\
0 & 52.0 & 0.0 & 3.2 \\
\hline
\end{tabular}

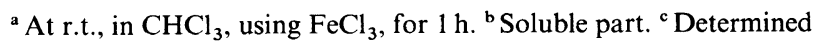
by GPC. for the 3-dodecylthiophene unit. The fraction of the 3-methylthiophene unit in the copolymers was calculated as the ratio of the signal intensities for the corresponding methyl protons.

In the case of copolymerization with largely different reactivity, a long polymerization time produces a mixture of copolymers with a multi-peak distribution because a more reactive monomer is polymerized faster and the monomer ratio in the polymerization solution changes. By a gel permeation chromatography analysis, the obtained copolymers were found to be homogeneous as can be seen by the single peak distribution in Figure 2 . This indicates that the ratio of copolymers in a polymer chain can be calculated from the ${ }^{1} \mathrm{H}$ NMR spectra. Figure 3 depicts the relation between the fraction of 3 -methylthiophene in the copolymerization feed and the copolymer. Except for the case of $60 \% 3$-methylthiophene feed, the data lay on a straight line with a slope of one. This implies that 3-methylthiophene is compatible with 3-dodecylthiophene for the copolymerization and that the 3-methylthiophene unit is attached to the polymer chain randomly.

The UV-VIS absorption spectra of the copolymer films are shown in Figure 4. The spectra have peaks around $500 \mathrm{~nm}$ assigned to the $\pi-\pi^{*}$ transition of the conjugated polymer main chains. The linear absorption coefficient and $\lambda_{\max }$ 's are affected by the contents of the copolymers. The coefficient and $\lambda_{\max }$ are plotted against the fraction of 3-methylthiophene for the copolymer in Figure 5. The $\lambda_{\max }$ is almost constant until the fraction of 3-methylthiophene exceeds $40 \%$, at which point $\lambda_{\max }$ decreases

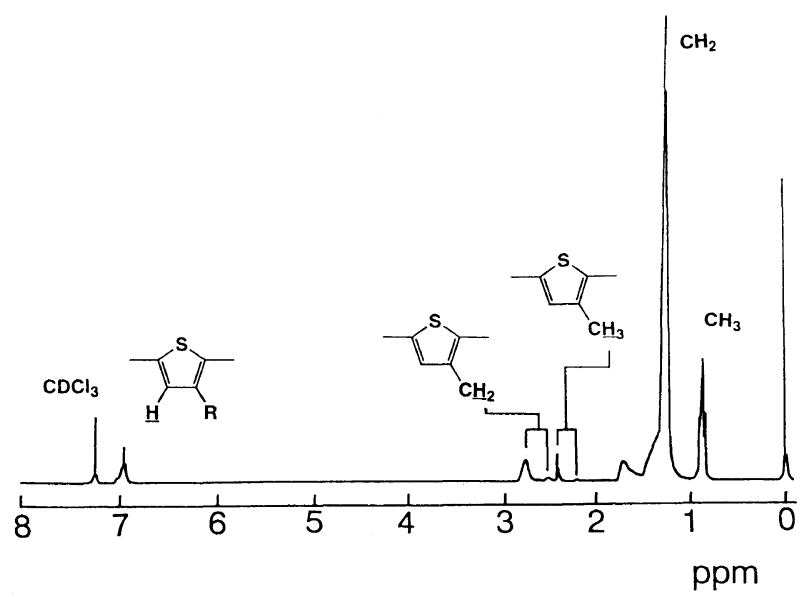

Figure 1. ${ }^{1} \mathrm{H}$ NMR spectrum of the obtained copolymer (3-methylthiophene fraction: $34.3 \%$ ).

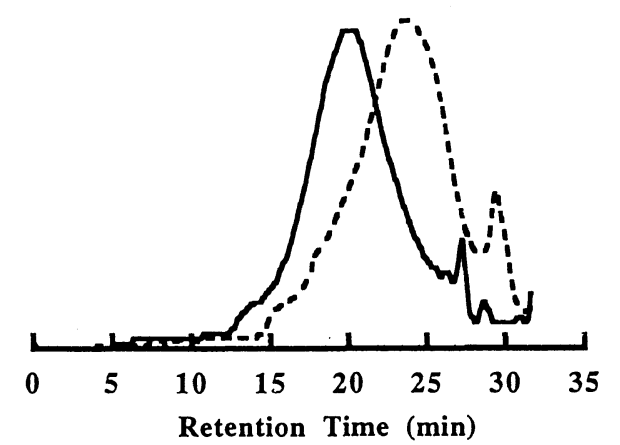

Figure 2. GPC curves of the copolymers. 3-Methylthiophene fraction: $0 \%(-), 34.3 \%(---)$. 


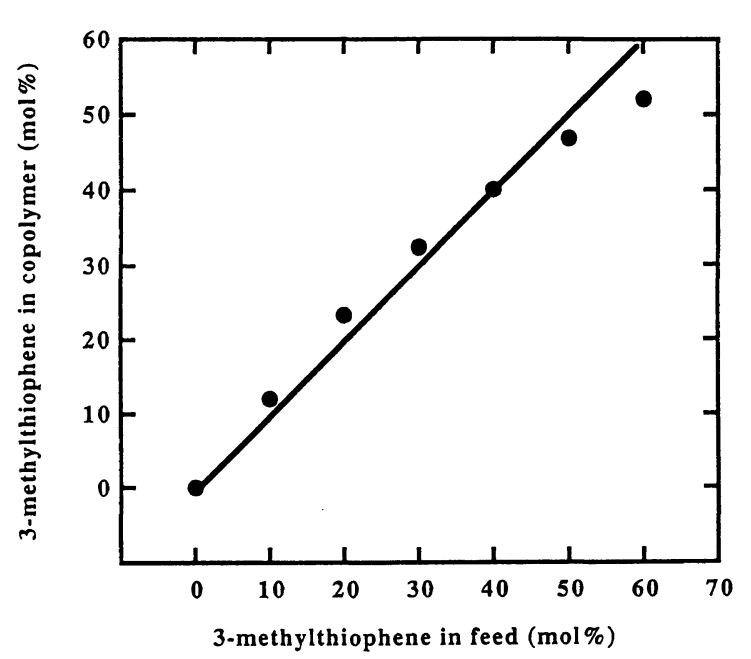

Figure 3. 3-Methylthiophene farction in the copolymers.

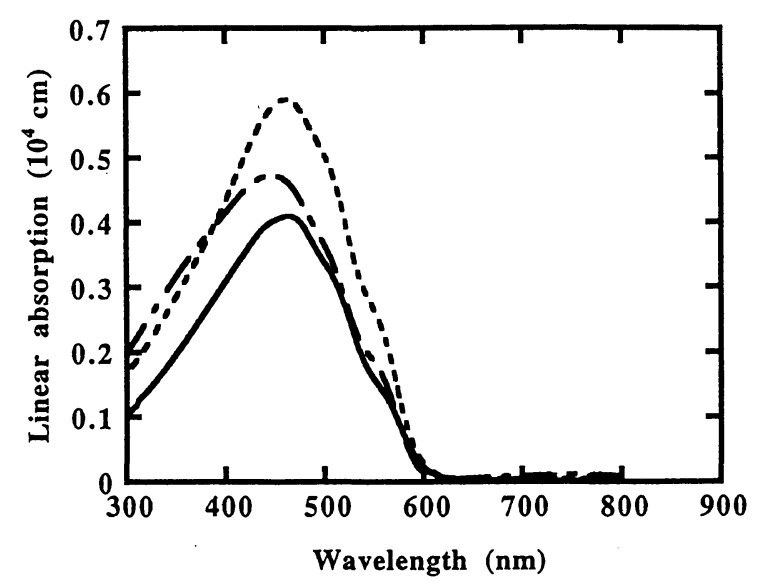

Figure 4. UV-VIS absorption spectra of the copolymers. 3-Methylthiophene fraction: $0 \%(-) ; 34.3 \%(--), 52.0 \%(---)$.

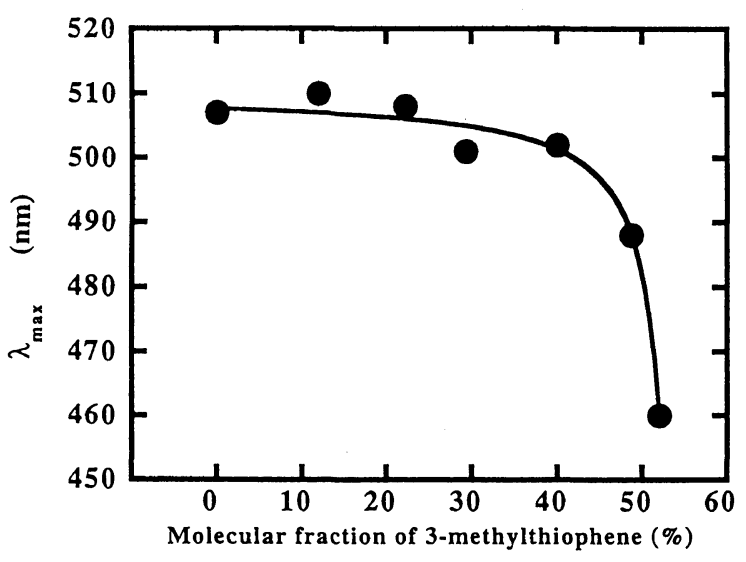

Figure 5. $\lambda_{\max }$ of the copolymers.

sharply. This indicates that the $\pi$-conjugation length is not changed by the copolymerization with 3-methylthiophene fractions lower than $40 \%$. The absorption coefficient curve has a peak around the fraction of $30 \%$ as shown in Figure 6 . The change of $\lambda_{\max }$ implies a decreasing of the $\pi$-conjugation length on the polymer main chain. ${ }^{20}$ The sharp blue-shift of $\lambda_{\max }$ can be explained as follows. The methyl group of the comonomer with different steric effect produces a defect on the $\pi$-electron conjugation states in the copolymer main

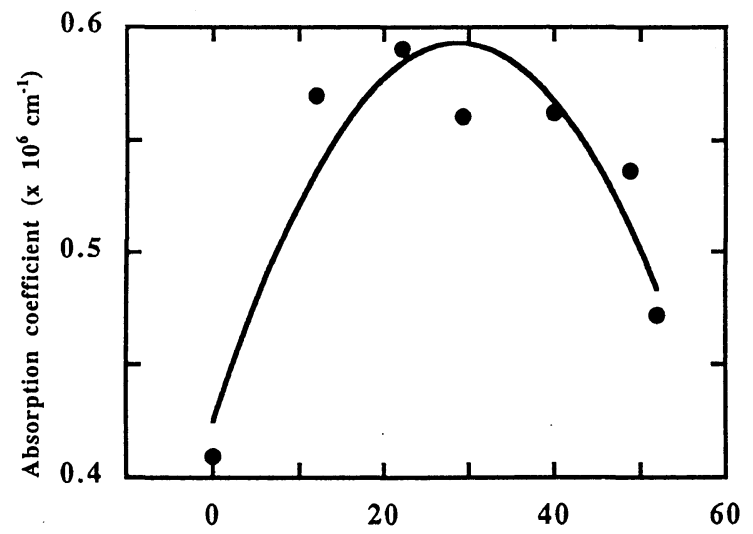

Molecular fraction of 3-methylthiophene (\%)

Figure 6. Linear absorption of the copolymers.

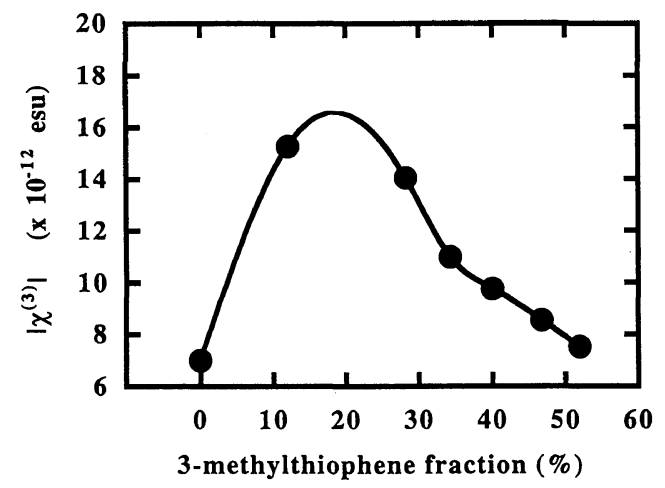

Figure 7. $\chi_{1111}^{(3)}(-3 \omega ; \omega, \omega, \omega)$ of the copolymers $\left(\lambda_{\omega}=1.907 \mu \mathrm{m}\right)$.

chains and reduces the $\pi$-conjugation length. On the other hand, mono-alkyl substituted polythiophenes have two modes of coupling, head-to-tail and head-to-head coupling. In the head-to-head coupling, the polymer main chains are twisted and the conjugation of the polymer is inhibited. As determined by the ${ }^{1} \mathrm{H}$ NMR study, the contents of this head-to-tail coupling is $10 \%$. Thus, the conjugation length of poly(3-alkylthiophene) is 10 repeating units. At low fractions of 3-methylthiophene (less than $30 \%$ ), the reduction of the $\pi$-conjugation by 3-methylthiophene unit is not pronounced and $\lambda_{\max }$ is not changed because the copolymers have already defect and the 10 repeating units length of the $\pi$-conjugation. When the fraction becomes higher than $30 \%$, the reduction of the $\pi$-conjugation by 3 -methylthiophene units becomes large enough that the blueshift of $\lambda_{\text {max }}$ is observed. The absorption coefficient is dominated by both the density of the main chains and the length of the $\pi$-conjugation. The introduction of 3-methylthiophene units to the copolymer decreases the density of the main chain and $\pi$-conjugation length. This trade-off relation results in the peak in the plot of the coefficient against the fraction of the 3-methyl monomer unit.

The nonlinear optical property of the copolymer was estimated by optical third harmonic generation. The absolute value of $\chi_{1111}^{(3)}\{-3 \omega ; \omega, \omega, \omega\}$ is plotted against the fraction of 3-methyl monomer. The third harmonic is $636 \mathrm{~nm}$ for the fundamental of $1907 \mathrm{~nm}$. This wavelength is in the tail of the absorption peak around $500 \mathrm{~nm}$. The $\chi_{1111}^{(3)}\{-3 \omega ; \omega, \omega, \omega\}$ of the copolymer is enhanced 
by three photon resonance and is also sensitive to the absorption around $500 \mathrm{~nm}$. The increase in the optical density of the copolymer enhance the $\chi^{(3)}$ of the copolymer and the blue shift of the absorption peak reduces it. Like the absorption coefficient, the curve for $\chi^{(3)}$ has a peak around the fraction of $20 \%$, and $\chi^{(3)}$ in the copolymer becomes three times that of poly(3-dodecylthiophene).

\section{CONCLUSION}

In summary, the copolymer with 3-dodecyl and 3methylthiophene was synthesized. The 3-methylthiophene is compatible to 3-dodecylthiophene in the copolymerization for fractions of 3-methyl monomer less than $40 \%$. The introduction of 3-methylthiophene unit into poly(3-dodecylthiophene) main chain causes a decrease of the $\pi$-conjugation in the main chain and increase of the NLO active chromophore because of the 3-methyl group in the comonomer. Shorter alkyl substitutions further reduces the $\pi$-conjugation length. The changes in the UV-VIS absorption spectrum and the nonlinear optical properties of the copolymer do not depend on the monomer fraction in a simple manner, rather there is a "trade-off" optimization point in the plot of $\chi^{(3)}$ versus 3-methylthiophene fraction. Finally, the $\chi^{(3)}$ is enhanced mostly around the fraction of $20 \%$.

\section{REFERENCES}

1. P. N. Prasad and D. J. Williams, Ed., "Introduction to Nonlinear Optical Effects in Molecules and polymers," Wiley-Interscience, New York, N.Y., 1991.

2. G. I. Stegeman, in "Nonlinear Optics," S. Miyata, Ed., Elsevier, Netherlands, 1992, p 337.
3. L. A. Hornak, Ed., "Polymers for Lightwave and Integrated Optics," Marcel Dekker, New York, N.Y., 1992, p 595.

4. W. S. Fann, S. Benson, J. M. J. Madey, S. Etemad, G. L. Baker, and F. Kajzar, Phys. Rev. Lett., 62, 1492 (1989).

5. C. Sauteret, J. P. Hermann, R. Frey, F. Pradere, J. Ducing, R. H. Baughman, and R. R. Chance, Phys. Rev. Lett., 36, 956 (1976).

6. T. Kanetake, K. Ishikawa, T. Hasegawa, T. Koda, K. Takeda, M. Hasegawa, K. Kubodera, and M. Kobayashi, Appl. Phys. Lett., 54, 2287 (1989).

7. R. Sugimoto, S. Takeda, H. B. Gu, and K. Yoshino, Chem. Express, 1, 635 (1986).

8. M. Sato, S. Tanaka, and K. Kaeriyama, Makromol. Chem., 188 , 1763 (1987).

9. S. Hotta, M. Soga, and N. Sonoda, Synth. Met., 26, 267 (1988).

10. W. E. Torruellas, D. Neher, R. Zanoni, G. I. Stegeman, F. Kajzar, and M. Leclerc, Chem. Phys. Lett., 175, 11 (1990).

11. H. Okawa, T. Hattori, A. Yanase, Y. Kobayashi, A. Carter, M Sekiya, A. Kaneko, T. Wada, A. Yamada, and H. Sasabe, Mol. Cryst. Liq. Cryst. Sci. Technol. Sec. B: Nonlinear Optics, 3, 169 (1992).

12. J. Messier, in "Nonlinear Optical Effects in Organic Polymers," J. Messier, F. Kajzar, P. N. Prasad, and D. Ulrich, Ed., NATO ASI Series E162, Kluwer, Dordrecht, 1989, p 47.

13. H. Okawa, T. Wada, and H. Sasabe, Mater. Res. Soc. Symp. Proc., 224, 263 (1992).

14. P. N. Prasad, in "Nonlinear Optical Effects in Organic Polymers," J. Messier, F. Kajzar, P. N. Prasad, and D. Ulrich, Ed., NATO ASI Series E162, Kluwer, Dordrecht, 1989, p 351.

15. U. Stamm, M. Taiji, M. Yoshizawa, T. Kobayashi, and K. Yoshino, Mol. Cryst. Liq. Cryst., 182A, 147 (1990).

16. T. Hattori, H. Okawa, T. Wada, and H. Sasabe, Opt. Lett., 17, 1560 (1992).

17. H. Okawa, M. Sekiya, J. Osawa, T. Wada, A. Yamada, H. Sasabe, and T. Uryu, Polym. J., 23, 147 (1991).

18. M. Leclerc, F. M. Diaz, and G. Wegner, Makromol. Chem., 90, 3105 (1989).

19. M. Sato, T. Shimizu, and A. Yamauchi, Synth. Met., 41, 551 (1991).

20. R. M. S. Maior, K. Hinkelmann, H. Eckert, and F. Wudl, Macromolecules, 23, 1268 (1990). 